INPLASY

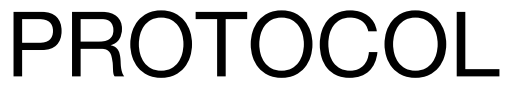

To cite: Guyatt et al. The effectiveness of physical exercise in management of stress and fatigue in people living with HIV/AIDS. Inplasy protocol 202140039. doi: 10.37766/inplasy2021.4.0039

Received: 07 April 2021

Published: 07 April 2021

Corresponding author: Guyatt GH

obinnacredible@gmail.com

Author Affiliation:

University Of Nigerian Enugu Campus

Support: UNCLE.

Review Stage at time of this submission: Preliminary searches.

Conflicts of interest: None declared.

\section{The effectiveness of physical exercise in management of stress and fatigue in people living with HIV/AIDS}

Guyatt, GH¹; Jayalath, JSS²; Thangavel, DN³ .

Review question / Objective: What is the effectiveness of exercise in the management of stress and fatigue in people living with HIV/AIDS, including those that are HAARTexperienced and HAART-naïve.

Condition being studied: HIV/AIDS patient.

Search strategy: The subject will be divided into the PICO framework. The keywords will be searched in PubMed to get their relative MeSH terms. The Boolean operators to be used are OR, AND NOT. Keywords and MeSH terms under POPULATION HIV/AIDS, HIV, AIDS, HIV patients, HIV/AIDs patients, HIV positive patients people living with HIV/AIDs, RNA Viruses, RETROVIRIDAE, HIV Infections, HIV seropositive patients. Keywords and MeSH terms under INTERVENTION Physical Activity, Exercise, Resistance training, Strength training, Exercise training, Training, Aerobic exercise, Endurance exercise, Weight lifting, Resistance exercise, Isometric Exercise. Keywords and MeSH terms under OUTCOME STRESS, MENTAL DISORDERS, TRAUMA, ACUTE, MENTAL SUFFERING, BURNOUT ,psychological STRESS, LIFE STRESS,FATIGUE, MENTAL FATIGUE, ASTHENIA, FRAILITY, LASSITUDE ,MUSCLE WEAKNESS ,COMPASSION FATIGUE, ALERT FATIGUE The BOOlean operators OR, AND and NOT OR will be used to join the respective keywords and MeSH terms in each of PIO AND will be used to join population, intervention and outcome NOT will be to search for HIV-1 instead of HIV-2 (HIV-1 NOT HIV-2).

INPLASY registration number: This protocol was registered with the International Platform of Registered Systematic Review and Meta-Analysis Protocols (INPLASY) on 07 April 2021 and was last updated on 07 April 2021 (registration number INPLASY202140039).

\section{INTRODUCTION}

Review question / Objective: What is the effectiveness of exercise in the management of stress and fatigue in people living with HIV/AIDS, including those that are HAART-experienced and HAART-naïve. 
Condition being studied: HIV/AIDS patient.

\section{METHODS}

Search strategy: The subject will be divided into the PICO framework. The keywords will be searched in PubMed to get their relative MeSH terms. The Boolean operators to be used are OR, AND NOT. Keywords and MeSH terms under POPULATION HIV/AIDS, HIV, AIDS, HIV patients, HIV/AIDs patients, HIV positive patients people living with HIV/AIDs, RNA Viruses, RETROVIRIDAE, HIV Infections, HIV seropositive patients. Keywords and MeSH terms under INTERVENTION Physical Activity, Exercise, Resistance training, Strength training, Exercise training, Training, Aerobic exercise, Endurance exercise, Weight lifting, Resistance exercise, Isometric Exercise. Keywords and MeSH terms under OUTCOME STRESS, MENTAL DISORDERS, TRAUMA, ACUTE, MENTAL SUFFERING, BURNOUT, psychological STRESS, LIFE STRESS, FATIGUE, MENTAL FATIGUE, ASTHENIA, FRAILITY, LASSITUDE, MUSCLE WEAKNESS, COMPASSION FATIGUE, ALERT FATIGUE The BOOlean operators OR, AND and NOT OR will be used to join the respective keywords and MeSH terms in each of PIO AND will be used to join population, intervention and outcome NOT will be to search for HIV-1 instead of HIV-2 (HIV-1 NOT HIV-2).

Participant or population: Adults with HIV/ AIDS.

Intervention: Physical exercise for patients with HIV.

\section{Comparator: No recent/current.}

Study designs to be included: Randomized controlled trial (RCTs).

Eligibility criteria: The participants: This literature review will include adult human participants aged $=\mathbf{1 8}$ years living with HIV.

Information sources: PubMed The Cochrane Library Pedro MEDLINE AMED CINAHL Ovid Embase.
Main outcome(s): Physical efficacy.

Additional outcome(s): Physical total efficacy.

Data management: Results of searches will be stored in EndNote and reviewed in coevidence, Titles and abstracts will be screened by one reviewer with $10 \%$ examined by a second reviewer. The full text will be retrieved for all records identified as eligible.Full text will be reviewed for eligibility by two reviewers in duplicate, with a third author consulted to resolve any discrepancies.Data will be extracted and entered into a pre-defined and piloted Microsoft Excel Database.Data will be extracted by one reviewer and independently checked by a second reviewer.Discrepancies will be identified and resolved through discussion ( with a third author where needed).

Quality assessment / Risk of bias analysis: The Cochrane risk of bias tool will be used to assess the risk of bias in Randomized Controlled Trials (https:// methods.cochrane.org/bias/resources.

Strategy of data synthesis: First, the question of the effectiveness of exercise would be answered, and thus all quantitative study results which examined the effect of this intervention will be presented, compared and pooled in an evidence table. Appropriate statistical techniques were used for each type of continuous (weighted mean differences if outcomes are consistent or standard mean difference if different outcomes are used, with $95 \% \mathrm{Cl}$ ) and divided variables (risk ratios, with $95 \% \mathrm{Cl}$ ). Characteristics of the retained studies will be sorted by year of publication and presented in a tabular form providing information relating to authors' references, sample size, age, setting, data collection format, outcomes, components of the intervention, component of the control, format and provider of the intervention, intervention and follow-up periods, and results The Analysis and presentation of results will be made in hierarchical order with the outcomes coming before the additional variables. 
Studies will be interpreted using narrative synthesis following the recommendation of the Centre for Reviews and Dissemination to explore the relationship and findings between and within the included studies (Akers et al. 2009).

Subgroup analysis: If the necessary data are available, subgroup analyses will be conducted with different comparators separately.

Sensitivity analysis: Sensitivity analysis is mainly used to evaluate the robustness of the primary outcome measures. The method is that removing the low-level quality study one by one and then merge the data to assess the impact of sample size, study quality statistical method, and missing data on results of meta analysis.

Language: English.

Country(ies) involved: Nigerian.

Keywords: Physical exercise, Stress and Fatigue, HIV/AIDS, Protocol, Systematic review.

Contributions of each author:

Author 1 - Guyatt GH - Conceptualization, Resources, Software.

Author 2 - Jayalath J.S.S - A systematic review of the effectiveness of strengthening exercise on HIV Positive patients.

Author 3 - Thangavel D.N - Data curation, Formal analysis, Supervision. 